ISSN: 0212-5374

DOI: http://dx.doi.org/10.14201/et2014321141159

\title{
DESARROLLO DE LA FORMACIÓN PERMANENTE DEL PROFESORADO EN LA PROVINCIA DE GUADALAJARA: UN ESTUDIO DE CASO
}

\author{
Development of permanent training of teachers \\ in the province of Guadalajara: a case study
}

\section{Développement de la formation permanente des enseignants dans la province de Guadalajara: une étude de cas}

\author{
Ricardo PIÑA SARMIENTO \\ CEIP "José Maldonado y Ayuso" (Mondéjar, Guadalajara). Correo-e: ricardopinasa@ \\ botmail.com
}

Recibido: 31-05-2013; Aceptado: 05-11-2013; Publicado: 30-03-2014

BIBLID [0212-5374 (2014) 32, 1; 141-159]

Ref. Bibl. RICARDO PIÑA SARMIENTO. Desarrollo de la formación permanente del profesorado en la provincia de Guadalajara: un estudio de caso. Enseñanza \& Teaching, 32, 1-2014, 141-159.

RESUMEN: El profesorado, una vez concluida su carrera, no puede contentarse con la Formación Inicial recibida. Del intento de adaptar el Sistema Educativo al devenir cultural y socioeconómico surge la necesidad de la Formación Permanente del Profesorado. Dicho modelo de Formación Permanente debe partir de una reflexión del profesorado sobre su práctica docente. Con este artículo hemos querido analizar el modelo de Formación Permanente del Profesorado en la Comunidad de Castilla-La Mancha, y concretamente en la provincia de Guadalajara, como factor de calidad en el desempeño de las funciones docentes de hoy en día. Para ello, hemos llevado a cabo un estudio de caso basado en una metodología descriptiva 
RICARDO PIÑA SARMIENTO

DESARROLLO DE LA FORMACIÓN PERMANENTE DEL PROFESORADO

EN LA PROVINCIA DE GUADALAJARA: UN ESTUDIO DE CASO

y cuantitativa, donde hemos procedido a describir no sólo las características de los CEP, sino también los cursos que se han desarrollado a lo largo de estos últimos años en estos centros (centrándonos fundamentalmente en los cursos relacionados con las TIC y la Educación Musical en Educación Primaria). Las conclusiones derivadas del estudio muestran cómo a lo largo de estos años ha habido una gran oferta de cursos, fundamentalmente relacionados con las TIC, pero una escasez de oferta formativa relacionada con la Música.

Palabras clave: formación, profesorado, formación permanente, CEP.

SUMMARY: One time finished his career, cannot be content with the initial training received. The attempt to adapt the education system to the cultural and socio-economic future the need arises for the permanent teacher training. This model of lifelong learning should start with a reflection of the teachers about their teaching practice. With this article we wanted to analyze the model of permanent teacher training in the community of Castilla-La Mancha, and specifically in the province of Guadalajara, as a quality factor in the performance of the educational functions of today. To this end, we have carried out a case study based on a descriptive and quantitative methodology where we proceeded to describe not only the properties of the CEP, but also courses that have been developed over the last years in these centres (focusing mainly on courses related to ICT and music education in Primary education). The conclusions of the study show how over the years there have been a variety of courses, mainly related to ICT, but a shortage of music-related course offerings.

Key words: training, faculty, continuing education, CEP.

RÉSUMÉ: Teachers Enseignants, une fois terminé sa carrière, ne peut pas être satisfait de la formation initiale reçue. La tentative d'adapter le système éducatif à l'avenir culturel et socio-économique, la nécessité se pose pour la formation des enseignants permanents. Ce modèle de l'apprentissage continu doit commencer par une réflexion des enseignants sur leur pratique pédagogique. Avec cet article, nous avons voulu analyser le modèle permanent formation des enseignants dans la communauté de Castilla-La Mancha et plus précisément dans la province de Guadalajara, comme un facteur de qualité dans l'exercice des fonctions éducatives d'aujourd'hui. À cette fin, nous avons mené une étude de cas basée sur une méthodologie descriptive et quantitative où nous avons procédé pour décrire non seulement les propriétés de la CBE, mais aussi des cours qui ont été développés ces dernières années dans ces centres (en se concentrant principalement sur l'éducation musicale dans l'enseignement primaire et cours liés aux TIC). Les conclusions de l'étude montrent comment au fil des ans il y a eu une variété de cours, principalement liés aux TIC, mais l'insuffisance de l'offre de cours liés à la musique.

Mots clés: formation, faculté, formation continue, CEP. 


\section{INTRODUCCIÓN}

Tello y Aguaded (2009: 31) afirman que la base fundamental de la Formación Permanente del Profesorado tiene una de sus principales razones de ser en la continua evolución de la sociedad y, consecuentemente, de la adaptación a ella de los sistemas educativos y métodos de enseñanza que puedan responder a las demandas sociales del momento. Ambos autores creen que la profesión del docente exige en igual medida una continua remodelación para que pueda cubrir con garantías los procesos de Enseñanza-Aprendizaje; su actividad profesional a lo largo de los años conduce al profesorado a implicarse en actividades de actualización docente.

De esta manera podemos afirmar que los profesores son determinantes para la calidad del Sistema Educativo. Su papel es central y todo lo que facilite su participación, motivación y preparación favorecerá, sin lugar a duda, el mejor funcionamiento de la enseñanza y la calidad del sistema educativo. El profesor Pérez Ferra (2009: 29) entiende como modelo más adecuado de formación permanente para adquirir y desarrollar competencias aquel que articula formación y experiencias equilibradamente en centros de formación.

Los Centros del Profesorado se han concebido desde su creación como sistemas de apoyo externo a los Centros Educativos, es decir, como un conjunto interactivo de dos o más personas y procesos con la misión común de proporcionar apoyo a dos o más Centros Educativos, entendiendo el apoyo como el proceso de asesoramiento o asistencia a la mejora de la escuela, pudiendo adoptar diversas modalidades (formación, consulta, información, provisión de materiales, etc.) en relación con las dimensiones, procesos y actividades de la mejora educativa, y con un carácter externo dada su localización fuera de los Centros Educativos.

El objetivo del presente trabajo es analizar cuál ha sido en los últimos años la Formación Permanente del Profesorado llevada a cabo en los distintos CEP de la provincia de Guadalajara (antes de su desaparición), centrándonos especialmente en conocer cómo ha sido dicha formación referida a la Música y a las TIC en la provincia objeto de estudio. También vamos a conocer y analizar la oferta formativa que el nuevo Centro Regional de Formación del Profesorado de Castilla la Mancha ha propuesto desde su aparición.

Añadir también, aunque no se incluirá dentro de nuestro estudio, que de forma paralela a los CEP existen otras instituciones, como las universidades, los sindicatos y otras entidades sin ánimo de lucro organizadoras de actividades de formación, que proponen su oferta formativa de acuerdo con los contenidos establecidos como complementarios para su incorporación al Plan Regional de Formación Permanente del Profesorado de Castilla-La Mancha. 


\section{DeSARROLLO DE LA EDUCACIÓN PERMANENTE DEL PROFESORADO}

En el Preámbulo de la LOE se dice que la Formación Permanente del profesorado es un derecho y una obligación del profesor, así como una responsabilidad de las administraciones educativas y de los propios Centros Educativos. En los artículos 102 y 103, se explicita este principio y así se establece que periódicamente el profesorado deberá realizar actividades de actualización científica, didáctica y profesional en los centros docentes, en instituciones formativas específicas y en las universidades, debiéndose planificar actividades de formación diversificadas y gratuitas.

La labor del docente no es sencilla. Debe actualizar sus conocimientos científicos, reflexionar sobre los cambios culturales que se producen, analizar el contexto y las características del centro en el que va a desarrollar su trabajo, valorar las posibilidades de aprendizaje de sus alumnos y adaptar a ellos sus objetivos educativos. Estas dificultades propias del trabajo habitual de los docentes aumentan cuando se pone en marcha un proceso de reforma educativa. Es preciso, entonces, aprender nuevos conceptos y técnicas, desarrollar nuevas habilidades didácticas y realizar un mayor esfuerzo para dar respuesta a las demandas que la sociedad está planteando.

La escuela, por lo tanto, y siguiendo a Tello y Aguaded (2009: 32), no puede quedarse de los cambios sociales, sino que debe adaptarse a ellos; más aún, debería ir por delante, puesto que el cambio social debe ser planteado por los nuevos ciudadanos, ya que en sus manos está el entramado futuro social. Para ello, afirman los mismos autores, los programas de Formación del Profesorado deben estar en consonancia directa con la labor que los docentes tienen que desarrollar, de manera que tengan una repercusión positiva en la mejora de la calidad de la enseñanza.

La Formación del Profesorado es un derecho y un deber de todos los docentes, y una responsabilidad de las administraciones educativas que debe abordarse como una permanente adaptación a la renovación que precisa la educación del futuro. Como tal adaptación, requiere tener en cuenta las necesidades sociales más relevantes, así como la presencia de ciertos obstáculos que deben irse solventando poco a poco, sin que ello suponga un cambio demasiado brusco que pudiera provocar inseguridad, malestar e intranquilidad entre el profesorado.

Santos Guerra (2010: 195) afirma que su propuesta para realizar una Formación Permanente que tenga carácter profesionalizador pasa por la investigación de los profesionales sobre su propia práctica. Es una investigación que está unida inextricablemente con la formación y con la innovación.

Para Marqués Graells (2008: 5), la Formación Permanente debe estar graduada. Si se dispone de los recursos necesarios en el aula, con unos conocimientos básicos sobre TIC (editor de textos, navegación por Internet, e-mail) y conociendo los modelos didácticos sencillos con apoyos TIC, es posible desarrollar numerosas actividades innovadoras de aprendizaje en el aula. Y éste debe ser el punto de 
partida para los profesores que se inician, dejando la realización de completos cursos presenciales u on-line para una segunda etapa para los que quieran saber más y especializarse en algunos aspectos.

En cualquier caso, el mismo autor cree que las principales modalidades de acceder a la formación por parte del profesorado en activo son las siguientes:

- Presencial en centros de profesores u otras instituciones.

- Cursos on-line.

- Presencial en el propio centro (cursos, seminarios...). La mejor para los profesores que empiezan. Desarrollada en forma de cortos seminarios mensuales en los que se da formación práctica con sencillos modelos didácticos para que los apliquen inmediatamente en las aulas, y donde también se van comentando las dificultades y ventajas que los profesores van encontrando al hacerlo.

- Formación recibida a partir de la interacción con otros colegas en comunidades virtuales de profesores y consulta de web/blogs docentes.

- Asistencia a Jornadas y Congresos.

- Participación en investigaciones, en colaboración con la Universidad.

Los artífices de la Formación Permanente de los docentes, en general, y dentro de la provincia de Guadalajara son diversos. Principalmente a los Centros del Profesorado se les ha encomendado esa labor. Además, existen otras entidades que vienen desarrollando programas formativos de interés para enseñantes en activo tales como los sindicatos, universidades y asociaciones y federaciones del sector.

La formación habría que entenderla como un proceso continuo, no como una actividad puntual, en función de los medios tecnológicos y de las necesidades que le van surgiendo al profesor, sino una formación basada en el centro que permita crear comunidades de aprendizaje, una cultura de colaboración para el uso de las TIC y estructuras organizativas apropiadas.

\subsection{Reflexiones sobre la Formación Permanente del Profesorado}

Rosales López (2006: 28) afirma que en la actualidad no existen límites rígidos entre la Formación Inicial y la Permanente. De hecho, se considera la primera como una introducción a la segunda, que ha de estar presente y desempeñar un papel relevante a lo largo del ejercicio de la profesión.

Las Nuevas Tecnologías plantean un nuevo contexto de formación inicial, continua y permanente de los profesores. Esta realidad, según García Sempere (2011: 111), nos conduce hacia una necesaria renovación de la normativa sobre formación del profesorado; pero también nos ha de llevar a la búsqueda de una nueva cultura formativa que permitirá enseñar y entrenar a los estudiantes en nuevas estrategias para que sepan buscar informaciones, procesarlas, configurarlas y utilizarlas. 

EN LA PROVINCIA DE GUADALAJARA: UN ESTUDIO DE CASO

En esta línea, Sevillano García (2008: 172) afirma que las Nuevas Tecnologías y los nuevos medios que ellas hacen posibles plantean un nuevo contexto de formación inicial, continua y permanente de los profesores que debe contemplar, entre otras cosas, la existencia de la sociedad postindustrial, la vivencia en una sociedad de la información y el establecimiento de una sociedad en Red. Esta realidad nos lleva a la búsqueda de una nueva cultura formativa que permitirá enseñar y entrenar a los estudiantes en nuevas estrategias para que sepan buscar informaciones (fuentes actualizadas y diversas), procesarlas, configurarlas y utilizarlas para situaciones concretas y que las puedan transferir de forma flexible y polivalente a nuevas situaciones y a nuevos problemas.

La Formación Permanente del Profesorado debe atender a la demanda social pero sin olvidar las características de los docentes. Por eso, la Formación Permanente del Profesorado ha de estar en función del modelo de profesor que necesita el Sistema Educativo: un docente con un alto grado de capacidad de actuación, de reflexión sobre su práctica y de adaptabilidad a las situaciones conflictivas y cambiantes del aula y del contexto educativo.

Pero este modelo de profesor no puede estar al margen de la sociedad en la que vive, y, por tanto, la formación que reciba debe de tener en cuenta el momento social en el que se desarrolla. Asimismo, la escuela no puede ni debe ser ajena al aumento de tiempos de ocio debido al elevado nivel de paro como a la progresiva disminución de horas de trabajo, ni al cambio de la estructura familiar, ni a la progresiva integración de culturas diferentes, ni a la aparición de determinados movimientos sociales que surgen para contrarrestar algunas formas de violencia social, sobre todo en el ámbito urbano, ni tampoco debe olvidarse la influencia que los medios de comunicación ejercen en nuestra sociedad, que en muchos casos actúan en contra del desarrollo de una capacidad verdaderamente crítica.

La formación y el perfeccionamiento del profesorado en medios y materiales de enseñanza requiere por principio que sea una formación práctica; ahora bien, ello no debe confundirse con una formación meramente instrumental, sino una formación donde el profesor pueda simular estrategias de utilización concretas de medios, pueda diseñar y producir mensajes mediados, y pueda evaluarlos. Siempre reflexionando sobre las decisiones adoptadas y procurando que dichas reflexiones sean colaborativas entre diferentes profesionales de la enseñanza.

Todo ello es muy importante, pero de nada o de muy poco sirve planificar actividades no deseadas por el propio profesorado; pues si el objetivo fundamental de la formación es promover el desarrollo profesional, es necesario conocer las necesidades de los propios docentes, ya que, si se desea lograr un cambio educativo, éste ha de ser deseado por el colectivo al cual nos estamos refiriendo. Es importante la participación del profesorado en este punto, lo que supone un proceso de reflexión sobre sus problemas, dificultades o "carencias" formativas que les permitirá por una parte conocerlas, identificarse con ellas, ver las posibles relaciones existentes entre las mismas, tomar conciencia de que éstas son las 
que el grupo percibe como verdaderas, y establecer una priorización, lo que les ayudará como colectivo a sentirse cada vez más autónomos en la resolución de sus propios problemas. En este sentido, es muy importante la participación del profesorado en el proceso de planificación de actividades de formación, realizadas éstas fundamentalmente por los CEP, y que permiten conocer las demandas directas de profesores y profesoras. En realidad los CEP (Centros de Formación del Profesorado) son una institución cuya corta historia y con muchos vaivenes políticos muestra una estrecha vinculación con la política educativa española referida al perfeccionamiento del profesorado (Galiano León, 2009: 163).

De esta forma, se debe atender de una manera diversificada, teniendo en cuenta el perfil profesional, el momento educativo en que se encuentran, su propio nivel de desarrollo y su trayectoria profesional.

Sevillano García (2007: 10-13) afirma que en la actualidad, a la luz de las políticas de convergencia de la Unión Europea, las necesidades de formación de los maestros se pueden sintetizar en los siguientes aspectos:

- Movilidad e intercambios.

- Conocimiento de lenguas extranjeras.

- Tutorización del aprendizaje.

- Renovación y preparación mediante la formación continua.

- Incorporación de las TIC.

- Potenciar la pedagogía de la interculturalidad.

Para alcanzar estos logros es imprescindible la aplicación de inversiones cuantiosas y la financiación adecuada de las nuevas demandas. Las investigaciones de los especialistas en este campo deberían contemplar los siguientes principios:

- Se ha de enfocar la formación del profesorado como proceso.

- Integrar contenidos académicos y pedagógicos.

- Integrar teoría y práctica.

- Debe haber fuerte relación entre la formación recibida y las demandas educativas de la Comunidad Autónoma de España y de Europa.

- Ser consecuente con el carácter social y dinámico de la formación del profesorado.

- Se ha de trabajar con grupos reducidos que permitan la individualización, personalización e interiorización comunicativa.

- $\quad$ Principio de supervisión y control público.

- Principio de indagación y reflexión.

- Formación para la diversidad educativa, lingüística y cultural.

- Formación desde y para las Tic.

La incorporación de las TIC al desarrollo profesional de los docentes es un imperativo, ya no se reduce solo a que los docentes conozcan y manejen equipos tecnológicos. El actual desafío está, sobre todo, en conseguir que los profesores y 
futuros profesores reflexionen, investiguen y comprendan cómo los estudiantes de hoy están aprendiendo a partir de la presencia cotidiana de la tecnología.

Si pretendemos integrar las TIC en los distintos escenarios educativos, será preciso contar con un elevado número de profesores en dichos escenarios que conozcan cómo utilizar dichas tecnologías y que su empleo no se limite a algún grupo aislado de profesores con el espíritu característico de los pioneros.

Por tanto, se hace cada vez más imprescindible crear las condiciones favorables a través de medidas de apoyo a los profesores, donde se reconozca por parte de las autoridades académicas su esfuerzo y resultados.

De esta forma, Cabero Almenara (2004) señala que en la formación del profesorado en TIC se deben contemplar diferentes aspectos, que podemos sintetizar en los siguientes:

- La formación debe centrarse en aspectos más amplios que la mera capacitación en el hardware y software. Los aspectos se deben centrar en cuestiones sobre la enseñanza y el aprendizaje.

- Es necesaria una buena formación conceptual, que le haga incorporar conceptualmente lo aprendido sobre las Tic en esquemas conceptuales más amplios, y en su desarrollo profesional.

- La Formación del Profesorado en TIC no debe ser una actividad puntual y cerrada, sino que más bien debe ser un proceso continuo, en función de los medios tecnológicos y de las necesidades que le vayan surgiendo al profesor.

- $\quad$ No existe un único nivel de formación del profesorado, sino que los profesores pueden tener distintas competencias y capacidades en función de las necesidades que le vayan surgiendo.

- $\quad$ Es importante no sólo el manejo y la comprensión de las TIC, sino que el profesorado comprenda que las TIC le permiten hacer cosas diferentes y construir escenarios diferentes para el aprendizaje de los alumnos.

- Y no sólo es cuestión de cambiar los instrumentos, tecnologías y mecanismos que utilizamos para transmitir información, sino también de cambiar las cosas que hacemos, de hacer enfoques diferentes, y de crear entornos más ricos, interactivos y variados, para que los alumnos trabajen en los mismos.

Por lo que respecta a la Música, cada vez existe una mayor oferta de actividades lúdicas y/o académicas relacionadas con la Música. De esta forma, el profesorado de Música puede ampliar sus conocimientos y experiencia, no sólo a través de los diferentes cursos, talleres y/o Máster de especialización que imparten universidades, sindicatos u otros organismos sin ánimo de lucro, sino también a través de los diferentes estudios musicales conducentes a la obtención de distintos títulos de grado y estudios de Doctorado de las universidades españolas. La formación como profesores y profesoras en esta área debe orientarse hacia la elaboración de didácticas que integren de forma efectiva y eficaz las Tic como 
una herramienta más dentro del proceso de Enseñanza-Aprendizaje de la Música. Modelos metodológicos que, en definitiva, acerquen a los alumnos a diferentes fuentes de información y creación (medios audiovisuales, Internet, textos y otros recursos gráficos y sonoros) para aumentar su conocimiento y disfrute de la Música. De ahí que la introducción de las Tic en este contexto educativo pase necesariamente por que el profesorado tenga la formación necesaria para poder incorporarlas en el aula.

La formación debe, por tanto, constituirse en un proceso reflexivo que se realice a partir de las propias ideas de los docentes. La colaboración permanente entre la práctica escolar y la investigación rigurosa permitirá que la educación pueda dominar y explotar del mejor modo posible lo que unos consideran como instrumentos de esclavitud intelectual y otros, cada vez en mayor número, como una potente herramienta en manos del educador, que le permite preparar a los alumnos para enfrentarse al mundo actual.

\section{CONTEXTO DE LA INVESTIGACiÓN}

En Castilla-La Mancha (basándonos en el Decreto 78/2005, de 5 de julio, Docm 136, de 8 de julio de 2005), el Plan Regional de Formación Permanente del Profesorado se organiza en torno a los siguientes ejes de contenido:

- Desarrollo de los procesos personalizadores de la educación: modelos de educación inclusiva y de respuesta a la diversidad del alumnado; educación intercultural y educación en valores.

- Actualización científica y didáctica: lectura y escritura, matemáticas, educación plurilingüe, desarrollo de programas de aprendizaje permanente, la formación en TIC, el programa escuela 2.0, la educación infantil y los programas de cualificación profesional inicial.

- Desarrollo de la LOE y las competencias básicas: competencias, programación, organización, programación de tareas y metodologías cooperativas, evaluación diagnóstica, unidades de evaluación.

- Desarrollo de la competencia personal y profesional de los docentes: aspectos profesionales y personales del docente asociados a la salud laboral.

- Mejora de la calidad y la eficacia de los centros docentes: la evaluación de centro, la apertura de los centros, la gestión participativa de los centros; modelos de gestión de la convivencia y participación democrática, dirección escolar: liderazgo, coordinación, gestión, evaluación e innovación.

- Coordinación del Plan de Orientación de Zona.

- La identificación de las necesidades educativas del alumnado a través de la evaluación psicopedagógica y el dictamen de escolarización; la coordinación de los Equipos de Orientación y Apoyo; y la dinamización social y cultural para desarrollar actividades extracurriculares y planes de apertura en los centros docentes en los que intervenga (sólo para los CRAER). 
3.1. La Formación Permanente del Profesorado en Castilla la Mancha: los CEP de la provincia de Guadalajara

\subsubsection{Los Centros de Profesores; los CEP de la provincia de Guadalajara}

La provincia de Guadalajara ha contado, desde la aparición del Decreto 78/2005, el 8 de julio de 2005, y hasta finales del mes de febrero de 2012, al cerrarse los CEP provinciales y crearse un único CEP regional, con dos CEP, Centro de Profesores, ubicados en Guadalajara y Azuqueca, este último en sustitución de la localidad de Pastrana, y dos CRAE, Centro de Recursos y Asesoramiento a la Escuela Rural, en las localidades de Sigüenza y Molina de Aragón.

Cada uno de ellos ha tenido asignados un número concreto de Centros Educativos, tanto de Educación Primaria como Educación Secundaria, debiendo el profesorado realizar los cursos en su CEP correspondiente, aunque no se impide que un profesor realice un curso determinado en un CEP distinto al asignado a su Centro Educativo.

La composición y ámbito de actuación de estos Centros de Formación del Profesorado en la provincia de Guadalajara ha sido la siguiente:

- Dirección: El desarrollo de las tareas propias de la dirección y el asesoramiento en el desarrollo de las competencias profesionales en la organización y gestión de centros, en la participación de la comunidad educativa y en la práctica de actividades de educación no formal.

- Asesoría Lingüística: El desarrollo de las competencias lingüísticas y de la comunicación en español y en lenguas extranjeras con la perspectiva intercultural y plurilingüe.

- Asesoría científica: El desarrollo de las competencias matemáticas, científicas y tecnológicas.

- Asesoría de las Tecnologías Educativas: El desarrollo de las competencias en el uso didáctico de las Tecnologías de la Información y de la Comunicación.

- Asesoría de la Orientación y la atención a la diversidad: El desarrollo de las competencias de Orientación y la respuesta a la diversidad del alumnado, así como la coordinación del Plan de Orientación de la zona.

- Asesoría de desarrollo cultural y de expresión artística: El desarrollo de las competencias asociadas al conocimiento de la cultura y la expresión de ideas de forma creativa en una serie de medios de expresión, incluyendo la Música, como expresión corporal, la literatura y las artes plásticas. 
DESARROLLO DE LA FORMACIÓN PERMANENTE DEL PROFESORADO

EN LA PROVINCIA DE GUADALAJARA: UN ESTUDIO DE CASO

TABLA 1

Número de asesores en los CEP de la provincia de Guadalajara

\begin{tabular}{|c|c|c|c|c|}
\hline & \multicolumn{4}{|c|}{ NÚMERO DE ASESORES EN CADA CEP O CRAER } \\
\hline & Guadalajara & Azuqueca & Sigüenza & Molina \\
\hline Dirección & 1 & 1 & 1 & 1 \\
\hline Asesoría Lingüística & 3 & 2 & 1 & 1 \\
\hline Asesoría científica & 2 & 1 & 1 & 1 \\
\hline $\begin{array}{l}\text { Asesoría de las } \\
\text { Tecnologías educativas }\end{array}$ & 3 & 2 & 1 & 1 \\
\hline $\begin{array}{l}\text { Asesoría de la } \\
\text { Orientación y la atención } \\
\text { a la diversidad }\end{array}$ & 2 & 1 & $\begin{array}{l}2 \text { Orientadores y } \\
1 \text { PTSC (Profesor } \\
\text { Técnico de }\end{array}$ & $\begin{array}{l}2 \text { Orientadores y } \\
1 \text { PTSC (Profesor } \\
\text { Técnico de }\end{array}$ \\
\hline $\begin{array}{l}\text { Asesoría de desarrollo } \\
\text { cultural y de expresión } \\
\text { artística }\end{array}$ & 1 & 1 & $\begin{array}{l}\text { Servicios a la } \\
\text { Comunidad) }\end{array}$ & $\begin{array}{l}\text { Servicios a la } \\
\text { Comunidad) }\end{array}$ \\
\hline
\end{tabular}

\subsection{El actual Centro Regional de Formación del Profesorado de Castilla-La Mancha}

El 29 de febrero de 2012 se cerraron los diferentes Centros de Profesores ubicados en las distintas ciudades de Castilla-La Mancha, incluidos, claro está, los de la provincia de Guadalajara. Por el Decreto 59/2012, de 23/02/2012 se crea el Centro Regional de Formación del Profesorado de Castilla-La Mancha (CRFP), y se regula la estructura del modelo de Formación Permanente del Profesorado.

Con el Decreto 78/2005, de 5 de julio, se pretende la simplificación de la estructura de la red institucional de formación, la apuesta por las Nuevas Tecnologías y, por último, la predisposición de la modalidad de formación a distancia como sistema de impartición, todo ello tiene como consecuencia el ajuste de los costes de la Formación del Profesorado.

La estructura general de la Formación Permanente del Profesorado estará constituida por:

- $\quad$ El Centro Regional de Formación del Profesorado de Castilla-La Mancha.

- Las Unidades de Formación de los Servicios periféricos de las cinco provincias.

- Los Coordinadores de Formación existentes en todos los centros educativos de la región. 
4. Diseño METODOLÓGICO

\subsection{Objetivos}

Dada la importancia de la Formación Permanente del Profesorado en el día a día en el aula, en la actualización docente, etc., nos planteamos los siguientes objetivos con esta investigación:

- Conocer y analizar la oferta formativa (cursos, seminarios y grupos de trabajo) para el profesorado que los diferentes CEP de la provincia de Guadalajara han convocado a lo largo de estos últimos 4 años.

- Conocer cuáles y cuántos de estos cursos han estado relacionados con la Música y las Tic.

- Saber cuál es la nueva oferta formativa desarrollada por el CRFP.

- Diferenciar y contrastar la oferta formativa llevada a cabo por los CEP y el CRFP.

- Valorar las repercusiones en la formación del profesorado.

\subsection{Técnicas empleadas}

Se ha llevado a cabo un análisis descriptivo de los datos obtenidos, procedentes de variables cuantitativas que recogen el número de cursos ofertados por los distintos CEP de la provincia de Guadalajara en estos últimos 4 años, así como la oferta formativa que el nuevo CRFP ha realizado en el último curso. Dicho análisis lo hemos realizado interpretando datos representados en gráficos de barras, en los que se pueda apreciar claramente el comportamiento de la información recopilada.

Como fuentes de recogida de datos hemos utilizado dos vías:

- Equipos directivos de los distintos CEP para recabar la información necesaria, es decir, qué actividades formativas para el profesorado se han llevado a cabo en sus centros a lo largo de los siguientes cursos escolares: 2007-08, 2008-09, 2009-10 y 2010-11 (no se incluye el curso 2011-12 al desaparecer dichos centros y aparecer el nuevo CRFP). Los diferentes directores, excepto el CRAER de Sigüenza, donde no se nos facilitó ninguna información, nos remitieron a la secretaría del centro para la recogida de dichos datos.

- $\quad$ Las distintas webs de los CEP, CRAE, CRFP y la página web de Educación de la Junta de Comunidades de Castilla-La Mancha.

\subsection{Muestra}

Desde la aparición del Decreto 78/2005, el 8 de julio de 2005, y hasta finales del mes de febrero de 2012, al cerrarse los CEP provinciales y crearse un único CEP regional, la provincia de Guadalajara ha contado con dos CEP, Centro de 
Profesores (Guadalajara y Azuqueca, este último en sustitución de la localidad de Pastrana), y dos CRAER, Centro de Recursos y Asesoramiento a la Escuela Rural (Sigüenza y Molina de Aragón). Por lo tanto, la muestra para el desarrollo de nuestra investigación está constituida por:

- $\quad$ CEP de Guadalajara y CEP de Azuqueca de Henares.

- $\quad$ CRAER de Sigüenza y CRAER de Molina de Aragón.

- $\quad$ El nuevo CRFP (con sede en Toledo).

\section{RESUlTADOS}

Los resultados de los CEP y CRAER han sido los siguientes:

\section{FIGURA 1}

Número de cursos ofertados por los CEP de la provincia de Guadalajara

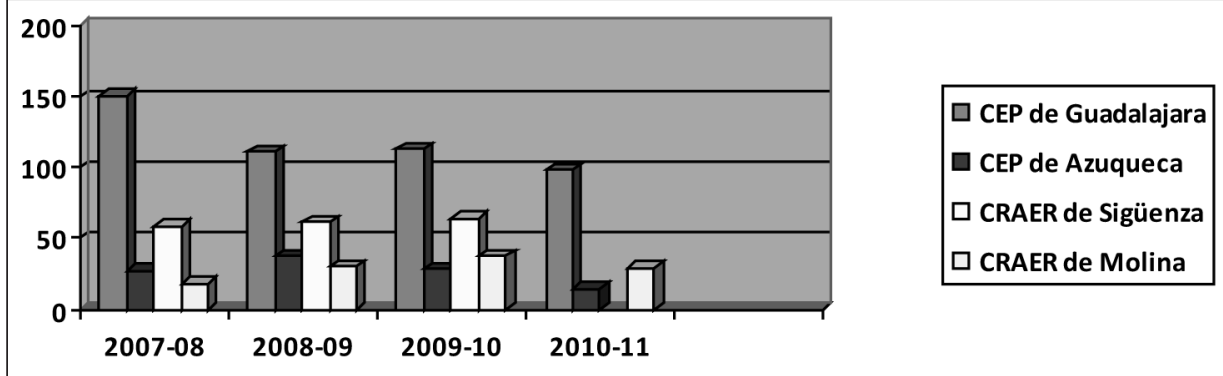

Como vemos, el Centro de Profesores de la capital es el que más cursos oferta al profesorado, al tener asignados mayor número de centros educativos, y por lo tanto mayor número de profesores. También observamos cómo se produce una disminución en la oferta de cursos de formación en cada año escolar, sobre todo en el CEP de la capital. Posiblemente por varios datos de vital trascendencia como son:

- Disminución de los profesores que realizan este tipo de cursos.

- Aumento de cursos online por otras instituciones.

- Escasez económica para convocar cursos (pago de kilometrajes al profesorado que asiste, pago a los ponentes, etc.).

- Posible desaparición de los CEP provinciales, lo que implica una reducción en la oferta de cursos. 


\section{FIGURA 2}

Número de cursos ofertados por los CEP de la provincia de Guadalajara relacionados con las TiC

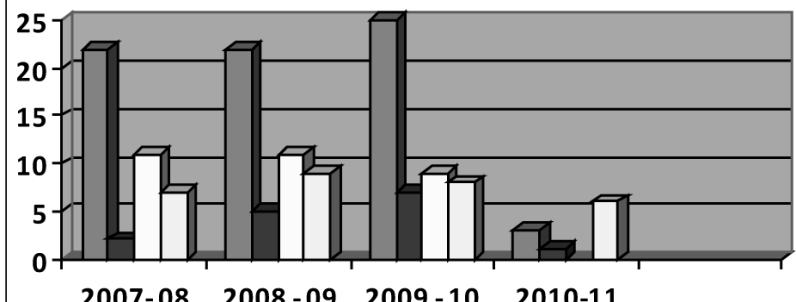

$\square$ CEP de Guadalajara CEP de Azuqueca $\square$ CRAER de Sigüenza $\square$ CRAER de Molina

Con respecto a la oferta formativa relacionada con las TIC, sigue siendo el Centro de Guadalajara el que más cursos ofrece, aumentando cada año el número de cursos ofertados (al igual que el Centro de Azuqueca), considerando, en mi opinión, la importancia y la actualidad del tema tratado. Aunque durante el curso 2010-11 la disminución de este tipo de cursos es considerable. Hemos de comentar que, al final del curso 2009-10, todos los CEP han organizado un curso "obligatorio" para los tutores de 5. y 6. de Educación Primaria y especialistas que impartan sus clases en ese curso, acerca del programa "Escuela 2.0" y, más concretamente, del programa "Smart Notebook 10".

\section{FIGURA 3}

Número de cursos ofertados por los CEP de la provincia de Guadalajara relacionados con la Educación Musical en Educación Primaria

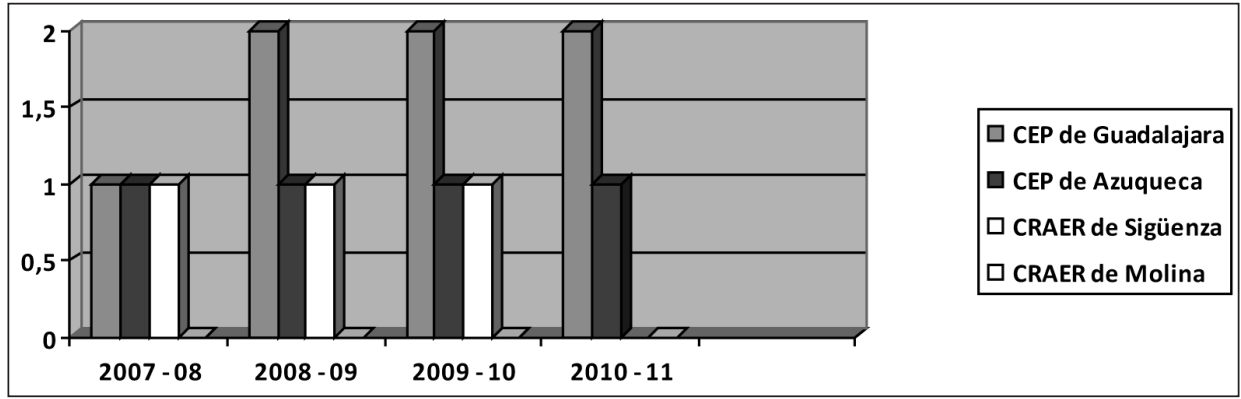

En cuanto a los cursos ofertados y relacionados con la Música en Educación Primaria, podemos observar que son muy escasos. Como posible "excusa", hemos de recordar que la especialidad de Educación Musical es impartida por un profesor, teniendo una sola hora lectiva semanal para cada curso, de ahí que con un solo profesor se cubra cada Centro Educativo o más (en el caso de los Centros Rurales 
Agrupados, CRA, e incluso Centros Educativos de la capital, Guadalajara, que al tener una sola línea, comparten profesorado de Música), de ahí que no sean muchos los profesores de Música los que tengan asignados cada Centro de Profesores, y sus demandas no tengan mucho efecto. Ese es el caso del CRAER de Molina, situado en la sierra de Guadalajara, con pueblos pequeños, ubicados en CRA, cuyo profesor es itinerante, impartiendo clases de Música a numerosos centros, que no ha organizado ningún curso de Música en estos últimos años.

Para concluir, y después de analizar todos los cursos que han ofertado los diferentes Centros de Profesores a lo largo de estos últimos años, decir que solamente el CEP de Guadalajara ha propuesto dos cursos en los que se relacionaba la Música con las TIC: curso escolar año 2007-08 titulado "Informática y composición musical aplicada al aula". También en el curso 2010-11 se realizó la actividad denominada "Hacemos Música y grabamos", pero no se realizó como tal curso en las instalaciones del CEP, sino que fue un Proyecto de Innovación Educativa propuesto y realizado por un Colegio Público de la capital. El resto de Centros de Formación no llevó a cabo ningún curso referente a dicho tema, posiblemente porque no ha habido una suficiente demanda por parte del profesorado de la zona concreta.

El profesorado prefiere la realización de cursos presenciales, que es una de las principales característica de los CEP, a los realizados on-line, ya que la especialidad de Música es práctica, y nada mejor que vivirla y presenciarla en los cursos, pero, por desgracia, no hay una gran oferta para el profesorado de Música. Por otro lado, y pasando de esta forma a los resultados de la investigación centrada en el nuevo CRFP, decir que el día 10 de abril de 2012 aparece publicada la oferta formativa a distancia para el profesorado castellano-manchego que se desarrollará en el curso 2011-12. En una sola edición se ofertaron 2600 plazas en un total de 16 cursos diferentes.

Los cursos ofertados fueron los siguientes:

- Competencias Básicas, una nueva forma de programar en el aula.

- Delphos Avanzado (para Equipos Directivos).

- Delphos Básico (para Equipos Directivos).

- Diseña tu web con Joomla.

- Cuadernia Avanzado.

- Cuadernia Básico.

- Escuela Digital.

- Estrategias y técnicas de investigación socioeducativa.

- Gestión económica de los centros docentes públicos no universitarios: Aplicación GECE 2000 (para Equipos Directivos).

- Gestión económica de los centros docentes públicos no universitarios: Marco Legal (para Equipos Directivos).

- Herramientas y recursos TIC aplicados a la educación.

- $\quad$ Las TIC en Primaria.

- $\quad$ Las TIC en Matemáticas. 

EN LA PROVINCIA DE GUADALAJARA: UN ESTUDIO DE CASO

- Google Earth y Google Maps.

- $\quad$ Powerpoint 2007.

- Respuesta educativa para el alumnado con TDAH (Déficit de atención e hiperactividad).

Como vemos, la temática principal de estos cursos desarrollados en este nuevo Centro de Formación se centra en las TIC, materia principal que el nuevo Gobierno de Castilla-La Mancha ha querido y quiere que se desarrolle en las aulas, comenzando, ante todo, desde la propia formación del docente.

\section{CONCLUSIONES}

Como resumen a lo expuesto anteriormente, podemos afirmar que las actividades realizadas por los distintos CEP, ya extinguidos, han sido numerosas y variadas en los últimos años; pero a pesar de ello, y centrándonos en las especialidades que nos interesan en este trabajo, éstas no han tenido la repercusión que se podría esperar de ellas, fundamentalmente las relacionadas con las Tic, las cuales, actualmente, son fundamentales para el correcto uso de los medios audiovisuales en el Centro Educativo.

Últimamente, la realización de Formación del Profesorado ha tomado un cariz diferente al que se iba desarrollando hace años. Actualmente, el profesorado se forma principalmente a través de dos vías:

- Los programas de Formación de los propios centros: donde el profesorado, sin salir de su lugar de trabajo, realiza esta formación a lo largo del curso escolar. Normalmente esta formación se lleva a cabo atendiendo a las necesidades del profesorado y sobre un centro de interés surgido en el propio Centro Educativo.

- Formación online o a distancia: podemos afirmar que actualmente es la formación preferida por el profesorado, quien libremente elige las actividades a realizar, atendiendo a sus gustos, intereses y preferencias. La realización de estas actividades es libre, siendo el propio profesorado quien organice su tiempo y trabajo para la realización de esta actividad.

Si éstas son las nuevas vías de formación del profesorado, también podemos afirmar que son dos de los motivos por los que los distintos CEP tuvieron una menor asistencia de profesorado en sus actividades. A estos motivos habría que añadir uno fundamental: la dificultad geográfica en la zona rural de la provincia de Guadalajara, donde hay profesorado (sobre todo en el área de Música) que realiza muchos kilómetros a lo largo del día para realizar su labor docente, y que le ocasiona dificultades (económicas, de tiempo, de organización personal, etc.) para desplazarse por las tardes a los CEP para formarse.

Parece necesario, de esta forma, recordar algunos aspectos que no por conocidos dejan de ser de notable actualidad en torno a las diversas iniciativas de Formación Permanente del profesor: 
DESARROLLO DE LA FORMACIÓN PERMANENTE DEL PROFESORADO EN LA PROVINCIA DE GUADALAJARA: UN ESTUDIO DE CASO

- Es necesario que el profesor participe tanto en la planificación como en el desarrollo de actividades de Formación Permanente que, en todo caso, deben estar precedidas por un adecuado diagnóstico de necesidades.

- $\quad$ Es imprescindible una cuidadosa selección de formadores de profesores.

- Es preciso fomentar la equidad y la igualdad de oportunidades.

- Es necesario facilitar la realización de actividades de Formación Permanente en los propios centros escolares.

- Es preciso utilizar la evaluación (de centros, de profesores, de alumnos, del sistema educativo) como instrumento de perfeccionamiento y no de represión o sanción pública o administrativa.

- $\quad$ En estos momentos se pone de relieve la necesidad de fomentar el reconocimiento social de la importancia de la función docente y de fomentar el apoyo al perfeccionamiento del profesor desde diversas perspectivas y organismos.

Por último, y refiriéndonos en este caso al nuevo CRFP, aún es pronto para sacar unas valoraciones y conclusiones acerca de su rendimiento, aceptación y operatividad. Pero lo cierto es que, al crearse un único centro regional, se "obliga" al profesorado a realizar su formación tanto en el mismo Centro Educativo donde esté destinado, como online.

El interrogante que nos planteamos para una futura investigación es saber si los profesores especialistas en la etapa de Educación Primaria (Educación Física, Música, Pedagogía Terapéutica, Audición y Lenguaje, Inglés), y que trabajan en zonas rurales, pueden actualizarse en su especialidad concreta a través del CRFP o lo han de hacer a través de otras instituciones, siendo, en algunos casos, actividades con un costo económico para el profesorado.

\section{REFERENCIAS BIBLIOGRÁFICAS}

Alonso García, C. M. y Gallego Gil, D. (1996). Formación del profesorado en tecnología educativa. En D. Gallego Gil (Coord.) (1996). Integración curricular de los recursos tecnológicos. Barcelona: Oikos-Tau.

Cabero Almenara, J. (2004). Formación del profesorado en Tic. El gran caballo de batalla. Revista Comunicación y Pedagogía. Tecnologías y Recursos Didácticos, 195, 27-31.

Castaño Garrido, C. y otros (2004). La utilización de las TIC en la Enseñanza Primaria y Secundaria obligatoria: necesidades de formación del profesorado. Barcelona: EDUTEC.

Cebreiro López, B. (1995). Los cambios en la Formación de los profesionales de la educación. Un análisis de la propia práctica. Revista Edutec 95-Materiales.

Decreto 78/2005, de 05-07-2005. Boletín Oficial de la Comunidad Autónoma de Castilla-La Mancha.

Decreto 59/2012, de 23/02/2012. Boletín Oficial de la Comunidad Autónoma de Castilla-La Mancha.

Díaz Mohedo, M. ํㅜ T. (2005). La formación del profesorado de Música ante la Convergencia Europea. Revista Electrónica Interuniversitaria de Formación del Profesorado (REIFOP) http://www.aufop.com/aufop/home. 
RICARDO PIÑA SARMIENTO

DESARROLLO DE LA FORMACIÓN PERMANENTE DEL PROFESORADO

EN LA PROVINCIA DE GUADALAJARA: UN ESTUDIO DE CASO

Fernández Martín, F.; Hinojo Lucena, F. J. y Aznar Díaz, I. (2002). Las actitudes de los docentes hacia la Formación en Tecnologías de la Información y la Comunicación (TIC) aplicadas a la Educación. Revista Contextos Educativos, 5, 253-270.

Fernández Muñoz, R. (2001). El profesor en la Sociedad de la Información y la Comunicación: nuevas necesidades en la Formación del Profesorado http://www.uclm.es/ profesorado/ricardo).

Galiano León, M. (2009) El asesoramiento profesional desde un centro de profesores. El análisis de la práctica. En L. M. Villar Angulo (Coord.). Creación de la excelencia en Educación Secundaria. Madrid: Pearson.

García Sempere, P. (2011). Formación del profesorado para la utilización de la cámara de vídeo digital en los centros de educación primaria. Revista Journal for Educators, Teachers and Trainers, 2, 107-117.

Gómez Alemany, L. y Romero Morante, J. (2009). Reformas educativas y formación Permanente del Profesorado en la última obra de Julia Varela: memoria y olvido. Profesorado, Revista de Currículum y Formación del Profesorado, 13 (1).

Gómez Alemany, I. y Vilar Monmany, M. (2003) Diez años de formación de maestros de música. Revista Eufonía, 28.

Gutiérrez Martín, A. (2008). Las TIC en la formación del maestro. "Realfabetización" digital del profesorado. Revista Interuniversitaria de Formación del Profesorado, 22 (3), 191-206.

Gutiérrez Martín, A. y otros (2010). La Formación de los futuros maestros y la integración de las TIC en educación: anatomía de un desencuentro. Revista de Educación, 352.

Lorenzo Yanes, A. I. (1999). La formación del maestro especialista en educación musical: análisis crítico del plan de estudios vigente. Revista Eufonía, 15.

Marcelo García, C. (2002). La formación inicial y permanente de los educadores. En Consejo Escolar del Estado. Los educadores en la sociedad del siglo XXI (pp. 161-194). Madrid: Ministerio de Educación, Cultura y Deporte.

Marqués Graells, P. (2000). Los docentes: funciones, roles, competencias necesarias, formación. Grupo de investigación DIM, Universidad Autónoma de Barcelona.

- (2008). La escuela del 2015. Las competencias TiC del docente. Contextualizadas desde el documento "Las competencias digitales de los docentes" http://dewey.uab.es/pmarques, .

Orden de 08-10-2008, de la Consejería de Educación y Ciencia de Castilla la Mancha. Boletín Oficial de la Comunidad Autónoma de Castilla-La Mancha.

Pérez Ferra, M. (2009). Capacidades para la cualificación profesional en el mercado de trabajo. En L. M. Villar Angulo (Coord.). Creación de la excelencia en Educación Secundaria. Madrid: Pearson.

Resolución de 30-09-2002 de la Dirección General de Coordinación y Política Educativa. Boletín Oficial de la Comunidad Autónoma de Castilla-La Mancha.

Rosales López, C. (2006). En torno a la Formación Permanente del Profesorado, motivos y procesos: reflexión sobre las características de la función docente y de la formación del profesorado. Revista Comunicación y Pedagogía, 212, 26-29.

Santos Guerra, M. A. (2010). La Formación del Profesorado en las instituciones que aprenden. Revista Redalyc (Red Interuniversitaria de Formación del Profesorado), 24 (2), 175-200. Zaragoza: Universidad de Zaragoza. http://www.redalyc.org/src/inicio/ArEdfRed.jsp?iCve=27419198009. 
DESARROLLO DE LA FORMACIÓN PERMANENTE DEL PROFESORADO

EN LA PROVINCIA DE GUADALAJARA: UN ESTUDIO DE CASO

Sevillano García, M. ․ L. (1998). Retos al profesor ante las nuevas tecnologías y medios de comunicación. Revista Profesorado, 2 (1), 35-57.

- (Coord.) (2002). Nuevas Tecnologías, medios de comunicación y educación. Formación Inicial y permanente del profesorado. Madrid: Editorial cCS.

- (Coord.) (2007). Investigar para innovar en enseñanza. Madrid: Pearson Prentice Hall.

- (2008). Investigar para innovar en enseñanza. Revista Religión y Cultura, LVI, 167-200.

Tello Díaz, J. y Aguaded Gómez J. I. (2009). Desarrollo profesional docente ante los nuevos retos de las tecnologías de la información y la comunicación en los centros educativos. Pixel Bit, Revista de Medios y Educación, 34, 31-47.

Torres del Castillo, R. M. $\underline{a}$ (1996). Formación Docente: claves de la reforma educativa. Publicado en: Nuevas formas de aprender y enseñar. Santiago: UnESCO-OREALC http:// www.fronesis.org).

Vilar i Monmany, M. y Gómez Alemany, I. (2005). De la Formación a la práctica: metodología para la evaluación de la Formación de Maestros de Música. Revista Interuniversitaria de Formación de Profesorado, 19 (2), 175-196. 